Red de Investigadores Educativos Chihuahua A.C.

\title{
Estado del arte sobre identidad docente: investigación de experiencias de profesores en formación y en ejercicio
}

IE Revista de Investigación Educativa de la REDIECH, 10(18), pp. 79-93. https://dx.doi.org/10.33010/ie rie rediech.v10i18.217

\section{(c) $(1)(\Theta$}

Esta obra está bajo licencia internacional

Creative Commons Reconocimiento-NoComercial 4.0. 


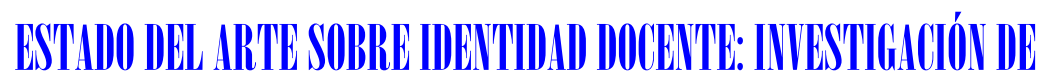

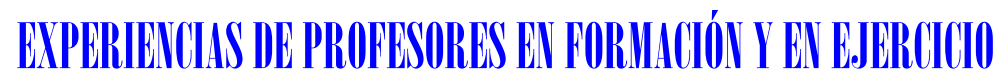

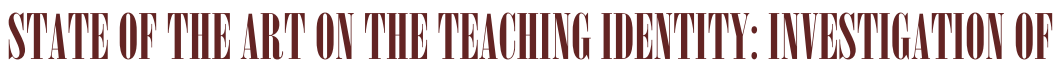

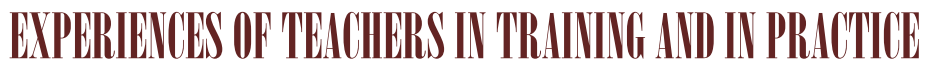

GAJARDO-ASBÚN Karen Paulina

Recepgión: Mayo 20 de 2018 | Aprobado para pUbucación: OCtuBre 14 de 2018

DOI: https://dx.doi.org/10.33010/ie_rie_rediech.v10il8.217

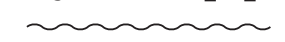

Resumen

El presente artículo tiene como objetivo dar cuenta del estado del arte de la investigación sobre la identidad docente focalizada en experiencias de profesores en formación y en ejercicio. Para ello se emplea análisis de contenido de tipo descriptivo, en el cual se analiza un total de 20 artículos publicados en la base de datos Scopus desde el año 2014 al 2017. Los resultados señalan que existen múltiples estudios a nivel mundial, investigación que se encuentra vigente y que busca comprender las aristas de la identidad en los diversos contextos que vive el educador. A partir de los resultados se espera contribuir con conocimiento de base para futuros estudios, los cuales den luces de lo que falta por investigar en este ámbito.

Palabras clave: IDENTIDAD DOCENTE, INVESTIGACIÓN, EXPERIENCIAS.

\begin{abstract}
The objective of this article is to give an account of the state of the art of research on teacher's identity focused on their experiences in training and in practice. For this, content analysis of a descriptive type is used, in which a total of 20 articles published in the Scopus database from 2014 to 2017 are analyzed. The results indicate that there are multiple studies worldwide, ongoing research that seeks to understand the edges

Karen Paulina Gajardo Asbún. Universidad Católica del Maule, Chile. Es profesora de educación básica mención lenguaje y magíster en política y gestión educacional. Actualmente es coordinadora del Programa de Atracción de Talentos en Pedagogía de la Universidad Católica del Maule. Su línea de investigación es formación docente y aprendizaje. Correo electrónico: kgajardoasbun@gmail.com. ID: http:/orcid.org/0000-0003-1386-461X.
\end{abstract}


of identity in the various contexts that the teacher's experiences. Based on the results, we expect to contribute basic knowledge for future studies, to provide insights into what remains to be investigated in this area.

Keywords: TEACHER IDENTITY, RESEARCH, EXPERIENCES.

\section{ITTRonicoriós}

\section{La investigación} centado en las últimas décadas en busca de comprender cómo la identidad puede influir en la práctica profesional y cómo puede llegar a afectar en los diversos contextos escolares. Identidad docente que envuelve una temática que contiene diversas aristas para su estudio, lo que fundamenta su nivel de investigación.

El concepto de identidad se vislumbra como una construcción individual y dinámica, resultado de diversos procesos de socialización respecto a un contexto sociohistórico. La identidad se va construyendo en la medida que el individuo experimenta, siendo parte de un sistema sujeto a transformaciones, ya sean en la organización económica, política y simbólica en las relaciones sociales (Dubar, 2002). El sujeto no está inmóvil, sino en constante cambio supeditado a las transformaciones que se van presentando en el contexto social, evolución que se ve reflejada en el actuar docente que emerge de la formación valórica, ideológica y por el contexto sociocultural en el que se sitúa.

El proceso de construcción de la identidad adquiere cambios en el tiempo, como plantea Quintriqueo (2010, p. 58); es referido a "acepciones diversas que por una parte se relacionan a las creencias y por otra a una concepción dinámica de la sociedad y la cultura"; por lo tanto, en cada sujeto la identidad se considera única y contingente a la vida personal y social, donde las experiencias de vidas son esenciales para tal construcción, caracterizada como un proceso evolutivo y de autoconocimiento.

El propósito del presente artículo es dar cuenta del estado del arte de la investigación sobre la identidad docente. Como esta temática posee múltiples perspectivas nos centramos en la búsqueda de artículos que expongan la identidad docente a través de vivencias de educadores que están en formación inicial o en ejercicio, apreciando el valor de las experiencias para la construcción de la identidad, de modo que se puedan identificar las tendencias en este campo y proponer futuros estudios a desarrollar en esta línea de investigación.

El análisis de las investigaciones busca responder las siguientes preguntas: ¿cuáles son los orígenes de las investigaciones?, ¿qué tipo de población se ha investigado?, ¿cuál es la metodología de investigación utilizada en los artículos?, ¿qué temas han sido objeto de estudio? y ¿qué recomiendan los autores a partir de sus hallazgos en investigación?

Para desarrollar la temática expuesta, en primera instancia se presenta la ruta metodológica asumida para la selección y el análisis de los artículos; posteriormente, se exponen los hallazgos a partir de las preguntas planteadas; para finalizar se 80 despliegan las conclusiones del análisis. 


\section{Netronolodil}

Para el presente estudio se opta por un diseño de análisis de contenido de tipo descriptivo. La búsqueda de documentos sobre la escritura de artículos de la identidad docente a través de experiencias de educadores que están en formación inicial o en ejercicio se realizó a partir de una revisión de la base de datos Scopus, una exploración de artículos escritos en inglés y español publicados en revistas de alto impacto. Para la indagación de los documentos se consideraron diferentes combinaciones de palabras clave. Por un lado, la palabra identidad, unida a las palabras profesor, docente y maestro. Cada expresión fue ingresada a la base de datos como motor de búsqueda junto con el filtro a los años de selección, en el cual se consideró desde el 2014 al 2017.

Al realizar la revisión de las fuentes bibliográficas de cada artículo se llegó al encuentro de otros documentos de la misma temática, por lo cual se utilizó la técnica de investigación cualitativa denominada "efecto bola de nieve" (Creswell, 2009). Esto quiere decir que un artículo seleccionado otorgó pistas para la búsqueda de otro a partir de sus referencias bibliográficas. El corpus de análisis estuvo compuesto por un total de 30 documentos en los cuales se realizó un segundo filtro para acotar la búsqueda, dirigido a artículos que estuviesen enfocados en la construcción de la identidad docente a través de experiencias en distintos escenarios, completando un total de 20.

En la tabla 1 se expone la identificación de los artículos seleccionados mediante el primer apellido del autor o autores y el año de publicación, así como el título y los objetivos que perseguían los trabajos seleccionados. Un primer análisis de esta información permitió identificar que los artículos de identidad docente están trabajados a partir de narrativas de estudiantes de pedagogía de distintos niveles y de profesores en ejercicio de diversas disciplinas. El gran número de documentos son estudios de caso y narrativas a través de entrevistas semiestructuradas; algunos de ellos relacionan la práctica con la formación y las experiencias de vida a la concepción de identidad. Para los análisis se relacionan los distintos actores, como formadores de profesores, estudiantes, docentes del sistema y sus influencias en la identidad del profesor. Dentro de las conclusiones se indica que el enfoque en la formación del profesorado no se centra en la identidad del maestro, por lo que existen vacíos en la formación respecto de una reflexión necesaria para comprender el impacto que posee en la profesión docente.

La identificación de las tendencias se realizó a partir de los 20 artículos científicos antes mencionados. Para examinar la información se diseñó e implementó una matriz de análisis con base en las preguntas planteadas en la introducción, como se observa en la tabla 2.

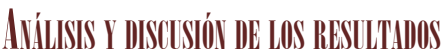

A continuación presentamos las tendencias a partir del año de la publicación, país donde se desarrollaron las investigaciones, características de los sujetos investigados 
Tabla 1. Identificación de las investigaciones por autor, año, título y objetivo

\begin{tabular}{lll}
\hline $\mathbf{N}$ & $\begin{array}{l}\text { Autor y } \\
\text { año de } \\
\text { publicación }\end{array}$ & Título del artículo \\
\hline
\end{tabular}

Exploración de la identidad docente desde una perspectiva

1 Bukor, 2014. holística: reconstrucción y reconexión personal y profesional.
Explorar la influencia de las experiencias personales y profesionales en el desarrollo de la identidad docente. La perspectiva holística en este artículo se refiere a la exploración de los profesores de idiomas de sus experiencias personales y profesionales con el uso de procesos de pensamiento consciente/racional e intuitivo/ tácito.

Investigar cómo las identidades docentes se construyen discursivamente en el curso de la formación docente y bajo la influencia de la estructura social, basándose en ideas de comunidades de práctica y teoría del discurso crítico.

2 Gu y Benson, docentes de inglés: una 2014. investigación intercultural.

La identidad lectora de los maestros en formación como

3 Granado y Puig, 2015. componente de su identidad docente. Un estudio de sus autobiografías como lectores.

Buscar una mayor comprensión de la identidad lectora de 88 futuros profesores de educación primaria que se hallaban en su último curso de formación, a través de sus autobiografías lectoras.

Convertirse en un educador a

Kenny, $\quad$ través de las artes: formando

4 Finneran y

Mitchell, 2015 . e informando la identidad profesional de los maestros emergentes.

\begin{tabular}{|c|c|c|c|}
\hline 5 & $\begin{array}{l}\text { Uitto, } \\
\text { Kaunisto, } \\
\text { Syrjälä y } \\
\text { Estola, } \\
2015 \text {. }\end{array}$ & $\begin{array}{l}\text { Verdades silenciadas: } \\
\text { dimensiones relacionales y } \\
\text { emocionales de la identidad de } \\
\text { un maestro principiante como } \\
\text { parte del contexto micropolítico } \\
\text { de la escuela. }\end{array}$ & $\begin{array}{l}\text { Comprender la identidad docente a través de un } \\
\text { enfoque narrativo, mediante historias basadas en } \\
\text { debates grabados en grupos de pares de } 11 \text { maestros } \\
\text { finlandeses. Los resultados ilustran cómo la identidad } \\
\text { de un maestro principiante se construye a través de diversas } \\
\text { relaciones emocionalmente significativas. }\end{array}$ \\
\hline 6 & $\begin{array}{l}\text { Pennington } \\
\text { y Richards, } \\
2015 \text {. }\end{array}$ & $\begin{array}{l}\text { Identidad del maestro en } \\
\text { la enseñanza del lenguaje: } \\
\text { integración de factores } \\
\text { personales, contextuales y } \\
\text { profesionales. }\end{array}$ & $\begin{array}{l}\text { Analizar las nociones de la identidad del profesor, cómo } \\
\text { se relacionan con las características específicas de la } \\
\text { enseñanza de idiomas y cómo la identidad del profesor } \\
\text { puede evolucionar o ser desarrollado a través de la } \\
\text { experiencia. }\end{array}$ \\
\hline 7 & $\begin{array}{l}\text { Quilaqueo, } \\
\text { Quintriqueo } \\
\text { y Riquelme, } \\
2016 .\end{array}$ & $\begin{array}{l}\text { Identidad profesional docente: } \\
\text { práctica pedagógica en contexto } \\
\text { mapuche. }\end{array}$ & $\begin{array}{l}\text { Explorar el proceso de construcción de identidad de los } \\
\text { docentes de Pedagogía Básica Intercultural que realizan su } \\
\text { actividad profesional en contexto mapuche, describiendo } \\
\text { las principales características con las que los mismos } \\
\text { protagonistas definen su identidad profesional docente. }\end{array}$ \\
\hline 8 & Izadinia, 2016. & $\begin{array}{l}\text { El desarrollo de la identidad } \\
\text { profesional de los profesores en } \\
\text { práctica y el rol de los maestros } \\
\text { mentores. }\end{array}$ & $\begin{array}{l}\text { Examinar los cambios en la identidad profesional de ocho } \\
\text { docentes y los factores que contribuyen a dichos cambios } \\
\text { durante una práctica de bloque de cuatro semanas. }\end{array}$ \\
\hline 9 & $\begin{array}{l}\text { Avraamidou, } \\
2016 .\end{array}$ & $\begin{array}{l}\text { Historias de sí mismo y de la } \\
\text { ciencia: la identidad de los } \\
\text { maestros de primaria antes del } \\
\text { trabajo a través del tiempo y en } \\
\text { todos los contextos. }\end{array}$ & $\begin{array}{l}\text { Explorar la naturaleza de dos identidades de docentes } \\
\text { primarios para la enseñanza de la ciencia y las experiencias } \\
\text { que impactaron su desarrollo a través del tiempo y en todos } \\
\text { los contextos. }\end{array}$ \\
\hline
\end{tabular}

Examinar la experiencia de los estudiantes y su formación de identidad dentro de un módulo de educación artística (que involucra música, drama y artes visuales) en un programa inicial de formación docente.
Comprender la identidad docente a través de un

Analizar las nociones de la identidad del profesor, cómo puede evolucionar o ser desarrollado a través de la

Explorar el proceso de construcción de identidad de los actividad profesional en contexto mapuche, describiendo las principales características con las que los mismos Examinar los cambios en la identidad profesional de ocho docentes y los factores que contribuyen a dichos cambios Explorar la naturaleza de dos identidades de docentes primarios para la enseñanza de la ciencia y las experiencias su desarrollo a través del tiempo y en todos 


\begin{tabular}{|c|c|c|c|}
\hline 10 & $\begin{array}{l}\text { Smilgiene, } \\
2016 .\end{array}$ & $\begin{array}{l}\text { La perspectiva sociocultural } \\
\text { sobre el proceso de formación de } \\
\text { identidad docente. }\end{array}$ & $\begin{array}{l}\text { Investigar el proceso de formación de identidad de siete } \\
\text { tutores no nativos que trabajan en un entorno terciario en el } \\
\text { Reino Unido para examinar los factores contextualizados } \\
\text { que moldean sus identidades. }\end{array}$ \\
\hline 11 & Elías, 2016. & $\begin{array}{l}\text { La construcción de identidad } \\
\text { profesional en los estudiantes } \\
\text { del profesorado de educación } \\
\text { primaria. }\end{array}$ & $\begin{array}{l}\text { Investigar los procesos de la construcción de la identidad } \\
\text { del maestro mediante el análisis de las historias producidas } \\
\text { por los propios estudiantes. El estudio se enmarca en una } \\
\text { concepción de identidad como narrativa. }\end{array}$ \\
\hline 12 & $\begin{array}{l}\text { Anspal, } \\
\text { Eisenschmidt } \\
\text { y Löfström, } \\
2016 .\end{array}$ & $\begin{array}{l}\text { Encontrándome a mí mismo } \\
\text { como profesor: explorando la } \\
\text { configuración de las identidades } \\
\text { docentes a través de las } \\
\text { narrativas de los estudiantes. }\end{array}$ & $\begin{array}{l}\text { Explorar el desarrollo de la identidad profesional entre } \\
\text { los estudiantes docentes en un programa integrado de } \\
\text { formación docente de cinco años en Estonia. }\end{array}$ \\
\hline 13 & $\begin{array}{l}\text { Yuan y Burns, } \\
2017 .\end{array}$ & $\begin{array}{l}\text { Desarrollo de la identidad } \\
\text { docente a través de la } \\
\text { investigación-acción: una } \\
\text { experiencia china. }\end{array}$ & $\begin{array}{l}\text { Explorar cómo dos profesores de idiomas construyeron y } \\
\text { reconstruyeron sus identidades profesionales a través de su } \\
\text { investigación de acción (AR) facilitada por investigadores } \\
\text { universitarios en China. }\end{array}$ \\
\hline 14 & Yazan, 2017 & $\begin{array}{l}\text { "Simplemente me hizo ver } \\
\text { el lenguaje de una manera } \\
\text { diferente": negociación de } \\
\text { identidad de los candidatos } \\
\text { docentes de ESOL a través de } \\
\text { cursos de educación docente. }\end{array}$ & $\begin{array}{l}\text { Investigar los casos de tres maestros de ESOL en un } \\
\text { programa de } 13 \text { meses de MATESOL centrándose en la } \\
\text { construcción discursiva, experiencial, negociada, disputada } \\
\text { y posicional de la identidad; conceptualiza el desarrollo de } \\
\text { la identidad como un componente integral del aprendizaje, } \\
\text { la práctica y el crecimiento de los docentes. }\end{array}$ \\
\hline 15 & $\begin{array}{l}\text { Nichols, } \\
\text { Schutz, } \\
\text { Rodgers y } \\
\text { Bilica, } 2017 .\end{array}$ & $\begin{array}{l}\text { La emoción de los primeros } \\
\text { maestros de carrera y las nuevas } \\
\text { identidades docentes. }\end{array}$ & $\begin{array}{l}\text { Desarrollar una comprensión de las conexiones entre } \\
\text { los episodios emocionales y las nuevas identidades de } \\
\text { maestros profesionales de los maestros de primer año. }\end{array}$ \\
\hline 16 & $\begin{array}{l}\text { Edwards y } \\
\text { Edwards, } \\
2017 .\end{array}$ & $\begin{array}{l}\text { Una historia de cultura y } \\
\text { enseñanza: la complejidad de la } \\
\text { formación de identidad docente. }\end{array}$ & $\begin{array}{l}\text { Explorar cómo un joven maestro de ciencias de secundaria } \\
\text { de Nueva Zelanda, educado en las escuelas maorí-medias } \\
\text { y luego en las escuelas medias inglesas de Nueva Zelanda, } \\
\text { desarrolla su identidad como maestro mientras navega por } \\
\text { una variedad de contextos y experiencias educativas. }\end{array}$ \\
\hline 17 & $\begin{array}{l}\text { Clarke, } \\
\text { Michell y } \\
\text { Ellis, } 2017 .\end{array}$ & $\begin{array}{l}\text { Dialéctica del desarrollo: } \\
\text { formación de identidad docente } \\
\text { en la interacción del ideal del ego } \\
\text { y del yo. }\end{array}$ & $\begin{array}{l}\text { Identificar la interacción entre las aspiraciones personales } \\
\text { de los docentes antes del servicio para su propia práctica e } \\
\text { identidad y sus percepciones de requisitos institucionales } \\
\text { más socializados y formalizados. }\end{array}$ \\
\hline 18 & $\begin{array}{l}\text { Fogle y Moser, } \\
2017 .\end{array}$ & $\begin{array}{l}\text { Identidades de profesores de } \\
\text { idiomas en el sur de los Estados } \\
\text { Unidos: transformando escuelas } \\
\text { rurales. }\end{array}$ & $\begin{array}{l}\text { Examinar las trayectorias personales y profesionales de los } \\
\text { profesores de idiomas en el servicio en entornos K-12 en } \\
\text { el estado de Mississippi para comprender mejor cómo los } \\
\text { participantes conceptualizan su práctica y sus roles en las } \\
\text { escuelas. }\end{array}$ \\
\hline 19 & $\begin{array}{l}\text { Nghia y Tai, } \\
2017 .\end{array}$ & $\begin{array}{l}\text { Profesores en formación, } \\
\text { desarrollo de la identidad durante } \\
\text { la práctica docente. }\end{array}$ & $\begin{array}{l}\text { Analizar dos narrativas de docentes antes del servicio } \\
\text { para resaltar el proceso de desarrollo de la identidad } \\
\text { docente durante su práctica. }\end{array}$ \\
\hline 20 & Jarauta, 2017. & $\begin{array}{l}\text { Construir la identidad } \\
\text { profesional de los maestros de } \\
\text { primaria durante su formación } \\
\text { inicial de docentes. El caso de la } \\
\text { Universidad de Barcelona. }\end{array}$ & $\begin{array}{l}\text { Comprender los procesos que los estudiantes experimentan } \\
\text { en la construcción de su identidad profesional de un } \\
\text { maestro de primaria. }\end{array}$ \\
\hline
\end{tabular}

Fuente: Elaboración propia. 


\begin{tabular}{llllllll}
\hline \multicolumn{10}{c}{ Tabla 2: Matriz de análisis } \\
\hline Año & $\begin{array}{l}\text { Autor/ } \\
\text { es }\end{array}$ & $\begin{array}{l}\text { País/ } \\
\text { es }\end{array}$ & Título & $\begin{array}{l}\text { Palabras } \\
\text { clave }\end{array}$ & Resumen & $\begin{array}{l}\text { Obje- } \\
\text { tivo }\end{array}$ & $\begin{array}{l}\text { Partici- } \\
\text { pantes del } \\
\text { estudio } \\
\text { menda- } \\
\text { ciones } \\
\text { del } \\
\text { autor/es }\end{array}$ \\
\hline
\end{tabular}

en las publicaciones, metodologías utilizadas por los investigadores, objetos de estudio y finalmente las recomendaciones de los autores. Algunas tendencias se describen en gráficas con su respectiva discusión y fueron analizadas a partir de la matriz.

\section{Origen de las investigaciones}

La tabla 3 representa la cantidad de artículos científicos revisados por año de publicación. Se puede observar que la temática ha sido constantemente abordada, "los últimos veinte años se ha desarrollado una línea de investigación sobre la identidad profesional de los docentes; sin embargo las temáticas, los enfoques y los propósitos de los investigadores son variados" (Elías, 2016, p. 337); por lo tanto, "la identidad profesional es un tema que ha traído el interés de muchos investigadores por décadas" (Nghia y Tai, 2017, p. 1), yaciendo una temática interesante de estudiar por su multiplicidad de aristas desde las cuales se puede profundizar.

A partir de la recogida de artículos, se puede

Tabla 3: Artículos científicos revisados por año de publicación

\begin{tabular}{ccc}
\hline Año & $\mathbf{N}$ & $\mathbf{\%}$ \\
\hline 2017 & 8 & $40 \%$ \\
\hline 2016 & 6 & $30 \%$ \\
\hline 2015 & 4 & $20 \%$ \\
\hline 2014 & 2 & $10 \%$ \\
\hline Total & $\mathbf{2 0}$ & $\mathbf{1 0 0 \%}$ \\
\hline
\end{tabular}

Fuente: Elaboración propia. concluir que los últimos años se ha presentado un mayor número de investigaciones que abordan la identidad docente mediante experiencias de educadores de formación inicial y en ejercicio, estudios que han sido publicados en revistas de alto impacto pertenecientes a la base de datos Scopus, existiendo un avance importante desde el 2014 con 10\%, hasta el 2017 con una representatividad del 40\%.

Estos datos demuestran la variedad de enfoques con los que se puede estudiar la identidad docente; por ejemplo, "se puede prestar especial atención a cómo modifican y desarrollan las identidades profesionales a través de la participación en múltiples comunidades de práctica, en diferentes ámbitos institucionales y contextos socioculturales (Yuan y Burns, 2017, p. 19), conceptos que pueden ser abarcados según el posicionamiento del autor y sus problemáticas de investigación según el contexto.

En la tabla 4 se evidencia el número total de artículos según el país donde se realizó la investigación y el continente de procedencia. En primer lugar, se observa que el mayor número de publicaciones (9) proviene de diferentes países de Europa; se destaca la producción de investigaciones provenientes de Reino Unido (3) y Es84 paña (2); le sigue América del Norte (4), con publicaciones de Estados Unidos (3) 


\begin{tabular}{|c|c|c|c|}
\hline País & No. publicaciones & Continente & \\
\hline Estados Unidos & 3 & & \\
\hline Canadá & 1 & América del Norte & 4 \\
\hline Argentina & 1 & & \\
\hline Chile & 1 & América del Sur & 2 \\
\hline Vietnam & 1 & & \\
\hline China & 2 & Asia & 3 \\
\hline Reino Unido & 3 & \multirow{6}{*}{ Europa } & \multirow{6}{*}{9} \\
\hline Estonia & 1 & & \\
\hline Irlanda & 1 & & \\
\hline Chipre & 1 & & \\
\hline Finlandia & 1 & & \\
\hline España & 2 & & \\
\hline Nueva Zelanda & 1 & \multirow{2}{*}{ Oceanía } & \multirow{2}{*}{2} \\
\hline Australia & 1 & & \\
\hline Total & \multicolumn{3}{|c|}{20} \\
\hline
\end{tabular}

Fuente: Elaboración propia.

y Canadá (1) y finalmente el continente de Asia (3) con experiencias desde China (2) y Vietnam (1); también se presentan publicaciones desde América del sur (2) y Oceanía (2).

Se deduce, por tanto, que hay un mayor número de publicaciones de autores angloparlantes, que tiene directa correlación con los artículos seleccionados según la revista, su procedencia y el idioma; sin embargo, la principal reflexión que se rescata de este apartado es que el tema de la identidad docente está abordado en la actualidad con representatividad en varios países del mundo.

\section{Cirracteristicias de los sujetos investigaidos en las publicacionnes}

La tabla 5 expone el número total de participantes de las investigaciones analizadas con su respectiva distinción entre los profesores en formación y los profesores del sistema escolar.

Existe una igualdad entre los artículos focalizados en investigaciones de profesores en formación (10) y profesores que se encuentran en ejercicio (10). En ambos se valora una riqueza a través de sus vivencias: "[...] vemos la identidad del maestro como

\begin{tabular}{lcc}
\hline \multicolumn{3}{l}{ Tabla 5: Sujetos investigados en las publicaciones } \\
\hline Profesores en formación & 10 & $50 \%$ \\
\hline Profesores del sistema & 10 & $50 \%$ \\
\hline Total & $\mathbf{2 0}$ & $\mathbf{1 0 0 \%}$ \\
\hline
\end{tabular}
narrativamente construida y reconstruida a través de historias [...]. Esta construcción se basa en los maestros dando sentido a sus experiencias" (Uitto et al., 2015, p. 163), por lo que es interesante conocer cómo se ha estudiado sus identidades.

Las etapas que vive cada educador son distintas y dependientes de cada contexto. Por una parte, los profesores en formación se encuentran en el inicio de su formación, por lo que las experiencias son fundamentales en su construcción de la identidad, la cual "se forma inicialmente antes de que los profesores ingresen a un programa de 
formación docente, con algunas imaginaciones idealistas, suposiciones o expectativas sobre la profesión" (Nghia y Tai, 2017, p. 2). Las investigaciones están enfocadas en observar la construcción de la identidad docente durante la formación inicial, su relación con las prácticas de intervención y la concordancia con sus experiencias de vida, las cuales le permiten decidir ser educador.

Respecto de los profesores en ejercicio, la identidad docente se va transformando por las experiencias que se viven en el día a día, en el contacto con los estudiantes, pares y por el contexto sociopolítico; como es de conocimiento, "los docentes viven en medio de diferentes historias colectivas sobre maestros, que inevitablemente forman una parte significativa de su identidad docente (Uitto et al., 2015, p. 164), lo que refleja que la relación con los demás influye en el pensamiento del profesor. Los artículos que abordan a los profesores en ejercicio centran la atención en la trayectoria de las experiencias personales y profesionales y cómo afectan en la construcción imperecedera de la identidad, en una variedad de contextos y prácticas educativas.

\section{Netodologías de investigación utilizizdlas en los irtículos}

Los investigadores manejan diversos diseños de investigación según su propósito y objeto de estudio. La tabla 6 da a conocer las metodologías de investigación utilizadas en las publicaciones analizadas, lo que permite dar una mirada global de los métodos empleados por algunos investigadores.

La tendencia señala que la metodología de investigación más utilizada por los investigadores es el estudio de caso (7), representado un 35\% del total de artículos. El estudio de caso, según lo definido por Creswell (2007), es una investigación de enfoque cualitativo en el que un investigador explora a lo largo de un tiempo, a través de una recopilación de datos detallada y profunda que involucra múltiples fuentes de información. En los artículos revisados se presentan diversos estudios de caso concernientes a docentes de distintas disciplinas y al seguimiento de docentes en formación universitaria y concerniente a maestros del sistema escolar.

En segundo lugar se encuentran las narrativas a través de entrevistas semiestructuradas (6), representando un 30\% del total. Demuestra que un gran número de investigadores escoge esta técnica para conocer en profundidad las identidades de los docentes; por lo tanto, las narrativas "son continuamente construidas, reflejan los contextos sociales en los que se crean e involucran ideologías dentro de los entornos sociales del individuo" (Taylor y Littleton, 2006). Por otra parte, el 25\% de las investigaciones se presenta a través de autobiografías (5); "a través de estas historias, los investigadores han intentado capturar toda la identidad. Consideramos

\begin{tabular}{|c|c|c|}
\hline Estudio de caso & 7 & $35 \%$ \\
\hline Narrativas a través de entrevistas & 6 & $30 \%$ \\
\hline Autobiografías & 5 & $25 \%$ \\
\hline Investigación educativa & 1 & $5 \%$ \\
\hline Revisión de literatura & 1 & $5 \%$ \\
\hline
\end{tabular}


la identidad del maestro como una historia compuesta de varias historias pequeñas" (Uitto et al., 2015, p. 165).

Otras metodologías utilizadas por los investigadores son investigación educativa (1) y revisión de literatura (1). Respecto de la investigación educativa, tiene como propósito el de construir conocimientos como una base para comprender y mejorar la formación inicial de docentes que se desempeñarán en contextos indígenas e interculturales (Rodríguez, Gil y García, 1996; Bisquerra, 2004; Quilaqueo, Quintriqueo y Riquelme, 2016). Finalmente se utilizó en un artículo la revisión de literatura (Pennington y Richards, 2016), una revisión de lo publicado sobre la identidad que ilustra extractos de narrativas de maestros.

Todas las técnicas expuestas de enfoque cualitativo exponen reflexiones profundas del proceso de construcción de la identidad y poseen un valor específico, ya que cada investigación se centra en distintos contextos y protagonistas, lo que amplía a un nivel macro la relevancia de este estudio en la actualidad.

\section{Temins que hitn sido objeto de investiganción}

El resultado de lo que se ha investigado en las publicaciones a partir de un análisis documental responde a la pregunta: ¿cómo se aborda la identidad docente en los artículos revisados? Para identificar el objeto de investigación se utilizó el análisis de contenido, método de investigación en el que Según Hernández Sampieri (2006, p. 357 ) "se efectúa por medio de la codificación, es decir, el proceso en virtud del cual las características relevantes del contenido de un mensaje se transforman a unidades que permiten su descripción y análisis preciso", categorías y subcategorías que se descubrieron a partir del análisis de los artículos.

En la tabla 7 se exponen las categorías y subcategorías clasificadas según el análisis de los resúmenes de los artículos estudiados, lo que nos permite obtener una mirada global de los enfoques abordados en las investigaciones.

En relación a la pregunta, la cual responde a cómo se aborda la identidad docente en los artículos revisados, al focalizarnos en el análisis de los resúmenes es posible visualizar que la categoría "autoconocimiento" (23) es la que tiene la mayor cantidad de recurrencias; es comprendida en este caso como un "proceso reflexivo y evolutivo en el cual cada educador adquiere noción de sus características personales que lo llevan a ser docente".

\begin{tabular}{lll}
\hline \multicolumn{3}{c}{ Tabla 7. Objeto de estudio de los artículos } \\
\hline Categoría & Subcategoría & Recurrencia \\
\hline \multirow{3}{*}{ Autoconocimiento } & Construcción & 9 \\
\cline { 2 - 3 } & Proceso de reflexión & 8 \\
\cline { 2 - 3 } & Desarrollo & 6 \\
\hline \multirow{2}{*}{ Experiencial } & Prácticas & 10 \\
\cline { 2 - 3 } & Experiencias & 8 \\
\hline \multirow{3}{*}{ Social } & Influencias & 10 \\
\cline { 2 - 3 } & Creencias & 6 \\
\cline { 2 - 3 } & Contextos & 6 \\
\hline
\end{tabular}

Fuente: Elaboración propia. 
Del análisis de los documentos se clasificaron subcategorías ordenadas de las más a menos recurrentes, tales como: "construcción" (9), subcategoría más concurrida entendida en este caso como "cimentación de la identidad a partir de experiencias vividas y su relación con las características personales y de contexto social que hacen al docente edificar constantemente su identidad"; una cita representativa es "la identidad del maestro, siendo una dinámica construcción, puede ser moldeada por una variedad de herramientas, tales como entornos sociales y culturales, políticos contextos, reformas escolares y rasgos personales" (Smilgienè, 2016, p. 67).

La segunda subcategoría con mayor número de recurrencias para esta respuesta es "proceso de reflexión" (8), la cual es definida como "fases sucesivas en la formación de la identidad docente en la cual se piensa sobre su actuar"; una cita identificada es "al énfasis que se coloca en los procesos de desarrollo de la identidad en el espacio y en todos los contextos, que esencialmente ilustra la historia de uno mismo" (Avraamidou, 2016, p. 60).

Finalmente, la última subcategoría es "desarrollo" (6), entendida en este caso como "el cambio que va adquiriendo el docente en la construcción de su identidad, en la cual integra aspectos experienciales"; una cita distintiva es "el desarrollo de la identidad del maestro podría ser una trayectoria que involucra diferentes experiencias de vida" (Nghia y Tai, 2017, p. 2).

Siguiendo con el análisis de cómo ha sido abordada la identidad docente, la segunda categoría más recurrente es "experiencial" (18), comprendida como "el valor de las experiencias para la formación de la identidad docente". La subcategoría más nombrada es "prácticas" (13), entendida en este caso como "experiencias en la cual el profesor en formación conoce y participa de diversos escenarios pedagógicos que le aportan a su formación"; una cita que expone este sentir es "teorizar enseñando y aprendiendo a enseñar en términos de identidad ofrece una forma de proporcionar espacio conceptual y práctico para cuestiones de autonomía, agencia y 'voz' en relación con la práctica profesional de los docentes" (Clarke, Michell y Ellis, 2017).

La segunda subcategoría de este segundo enfoque es "experiencias" (8), comprendido como "oportunidades para conocer y relacionarse con el sistema educativo, vivenciando diversos episodios que impactan en la identidad del profesor"; una cita representativa es "la identidad del maestro se desarrolla de manera que refleja la combinación única de cultura, contextos y experiencias que poseen" (Edwards y Edwards, 2016, p. 4).

La tercera categoría es "social", entendida en este caso como "características del medio social que influyen en la identidad del profesor". La subcategoría más repetida es "influencias" (10), comprendida como "efectos que producen otras personas en la identidad de cada profesor". Una cita representativa es "la identidad del maestro es una intrincada y enmarañada red de influencias e impresiones enraizadas en experiencias de vida personales y profesionales" (Bukor, 2014, p. 3).

La segunda subcategoría es "creencias" (6), en este caso entendida como "principios ideológicos de un individuo que forman parte de su identidad docente"; una cita de los resúmenes revisados que expone esta conceptualización es "la autoimagen y el sentido de identidad de una persona se basan en valores y creencias sobre cómo 
las personas deben conducir sus vidas y comportarse frente a otros" (Pennington y Richards, 2016, p. 3).

Finalmente, y con el mismo número de recurrencias, se encuentra la subcategoría "contextos" (6), comprendida como "escenarios que envuelven una situación y que impactan en el sujeto por lo que pueden intervenir en su identidad como profesor"; una cita que expone esta subcategoría es lo expresado por Jarauta (2017, p. 118):

El desarrollo de la identidad docente en los maestros en formación se asienta con fuerza en las rutinas y modelos pedagógicos de quienes fueron sus profesores en los niveles educativos precedentes, en vivencias significativas construidas con la familia durante la etapa infantil y en el contacto directo con el contexto escolar.

Por lo expuesto, se puede considerar que la identidad docente es abordada en los artículos revisados a través de trabajos que se focalizan en los propios docentes, pertenecientes a diversos contextos, estudios que mezclan teoría con datos cualitativos de las experiencias docentes. Tales artículos buscan entender la construcción y el desarrollo de la identidad docente a través de reflexiones en profundidad, donde se da vital importancia a las experiencias y a las prácticas de formación. También las investigaciones plantean la relevancia del contexto social, de las influencias y creencias que tienen los individuos para su concepción de la propia identidad.

\section{Recomendiaciones de los intureres}

La revisión de los artículos nos permitió conocer cómo los autores han abordado la identidad docente desde múltiples perspectivas, valorando las experiencias para efectuar los análisis de las investigaciones. Desde estos surgen en los apartados finales las conclusiones de los autores y las recomendaciones para futuros estudios, los cuales dan luces de qué falta por investigar en esa área.

Se concluye que la temática de la identidad docente es un tópico vigente por su impacto en el actuar docente. Respecto de las recomendaciones, se visualiza una tendencia respecto de investigar las identidades docentes focalizadas en grupos afines. Por ejemplo, profesores que imparten alguna disciplina específica, profesores en formación, en relación a módulos de aprendizaje que se imparten en la universidad, en las experiencias de las prácticas de formación, en las mentorías y en profesores del sistema con disímiles años de experiencia en el sistema escolar.

Los autores recomiendan profundizar en la reflexión y avanzar en investigación acerca de la formación de la identidad docente (Granado y Puig, 2015). Los investigadores ponen especial énfasis en que las futuras investigaciones puedan enfocarse en investigar diversos contextos para comprender el desarrollo de la identidad en cada uno de ellos y cómo estos afectan en las concepciones de los educadores; así, Yuan y Burns (2016, p. 19) señalan que los profesores en formación deben "prestar atención a cómo modifican y desarrollan sus identidades través de la participación en múltiples comunidades de práctica en diferentes instituciones y contextos socio culturales", dando valor a las experiencias previas a ejercer la profesión. 
Diversos autores señalan que los contextos afectan directamente la concepción de la identidad. Plantean que sería interesante hacer el contraste entre experiencias socioculturales desiguales. Además, se propone explorar cómo los individuos, como agentes reflexivos, construyen su identidad dada contextualmente con restricciones e influencias estructuradas del medio social (Gu y Benson, 2014). Junto con lo anterior, los autores sugieren que se investigue en relación a la identidad multicultural de los profesores (Gu y Benson, 2014; Smilgienè, 2016). Al mismo tiempo, se señala que los entornos familiares y personales pueden influenciar el desarrollo de la identidad (Nghia y Tai, 2017), por lo que se propone ampliar la investigación en ese campo.

Para futuros estudios es importante considerar las características personales en la formación de la identidad, las características que tienen en común los jóvenes que desean continuar estudios de pedagogía, considerar el aspecto emocional de los maestros en diferentes escuelas (Nichols et al., 2017). Se busca comprender la variedad de formas en que el contexto escolar y las características del estudiante influyen en la identidad docente.

\section{Concussoutes}

A partir de los artículos analizados es posible reconocer que el estudio de la identidad docente se desarrolla actualmente en todo el mundo y se le otorga vital importancia por el impacto que posee el docente en la sociedad. Conocer el proceso de construcción de la identidad resulta interesante, sobre todo para la búsqueda de mejoras en la formación inicial y en el legado que pueden dejar estas investigaciones, tanto para las decisiones de instituciones formadoras de profesores, como para políticas públicas relacionadas al profesorado.

La temática de la identidad docente centra su investigación en los profesores en formación y en los profesores que se encuentran en el sistema; su foco, por lo tanto, son las distintas etapas que vive el educador, relacionado a los contextos y experiencias de vida, dando valor a su rol en la sociedad. Por lo señalado, es relevante que se continúe con su investigación, pues el rol del docente es clave en la formación de una ciudadanía tolerante, responsable y solidaria, por lo que su investigación permite conocer los procesos de construcción y cómo estos pueden impactar en las generaciones de estudiantes.

Respecto de la metodología seleccionada por los investigadores, se utiliza el enfoque cualitativo, mediante el cual se exponen reflexiones profundas del proceso de construcción de la identidad. Para esto, Hernández, Fernández y Baptista (2010) señalan que en el desarrollo del proceso investigativo cualitativo se puede mencionar como principales conceptos al interaccionismo simbólico, las investigaciones etnográficas, investigación-acción, investigación participativa con teoría fundamentada, triangulación y diseños narrativos. Investigaciones que poseen un valor específico, ya que cada investigación se centra en distintos contextos y sujetos de estudio aportando al conocimiento.

Los diversos métodos utilizados por los investigadores en los artículos revisados, 90 narrativas, autobiografías y estudios de caso, buscan una aproximación al sujeto, 
quien cuenta su historia, la cual es interpretada por el investigador para conocer el fenómeno social en profundidad. En este aspecto se concluye que este tipo de investigación se pude situar bajo el paradigma participativo, paradigma que reúne el conocimiento en conjunto al protagonista, lo cual permite en la investigación ir concretando la interpretación del mundo.

Conocer el desarrollo de la identidad docente a partir del paradigma participativo accede al conocimiento desde el actor principal; así, "el paradigma participativo afirma que la gente no puede ser movilizada por una conciencia y un conocimiento que no sea suyo" (Loewenson et al., 2014, p. 21), por lo que se valora que el protagonista sea partícipe de toda la creación del conocimiento y tenga la autoridad de dar sus contribuciones al trabajo de estudio, proceso de investigación que busca crear el conocimiento desde una noción activa del sujeto.

Por todo lo expuesto, es importante que las identidades docentes se indaguen en la formación inicial de profesores a través de trabajos de autoconocimiento y reflexiones mediante un diálogo entre pares, sus familias y en las experiencias de práctica; por tal, corresponde que las universidades formadoras de profesores incluyan esta temática en sus mallas y otorguen una educación que les permita a los estudiantes de pedagogía madurar sobre su rol activo y transformador en la sociedad.

Esta reflexión genera inquietudes para próximas investigaciones. Resulta interesante ampliar el conocimiento de las identidades docentes en las instituciones formadoras. Conocer las identidades docentes de los académicos formadores de profesores permite aportar a la reflexión concerniente a la formación de profesores, como conocimiento directo para las universidades que imparten pedagogías y para las políticas educativas de formación inicial docente.

Por tanto, esta materia, al ser abordada en profundidad, contribuye en conocer el significado que le dan los profesores a la construcción de las identidades docentes, permitiendo observar la evolución y los cambios en los contextos a través del tiempo, además de reflexionar en relación al escenario pedagógico actual; "los trayectos que históricamente se han recorrido nos ayudan a repensar la actualidad" (Peiteado, 2010), siendo un aporte para la formación de las nuevas generaciones de profesores.

\section{ReFprenillis}

Anspal, T., Eisenschmidt, E. y Löfström, E. (2012). Finding myself as a teacher: Exploring the shaping of teacher identities through student teachers' narratives. Teachers and Teaching: Theory and Practice, 18(2), 197-216. doi 10.1080/13540602.2012.632268

Avraamidou, L. (2016). Stories of self and science: preservice elementary teachers' identity work through time and across contexts. Pedagogies: An International Journal, 11(1), 43-62. doi 10.1080/1554480X.2015.1047837

Bisquerra, R. (2004). Metodología de la investigación educativa. Madrid: La Muralla.

Bukor, E. (2014). Exploring teacher identity from a holistic perspective: Reconstructing and reconnecting personal and professional selves. Teachers and Teaching: Theory and Practice, 21(3), 305-327. doi 10.1080/13540602.2014.953819

Creswell, J. W. (2007). Qualitative inquiry and research design: Choosing among five approaches. Thousand Oaks, CA: Sage 
Creswell, J. W. (2009). Research design: Qualitative, quantitative, and mixed methods approaches. Thousand Oaks: Sage publications.

Clarke, M., Michell, M., \& Ellis, N. J. (2017). Dialectics of development: teacher identity formation in the interplay of ideal ego and ego ideal. Teaching Education, 28(2), 115-130. doi 10.1080/10476210.2016.1211631

Dubar, C. (2002). Crisis de las identidades. La interpretación de una mutación. Barcelona: Ediciones Bellaterra S.L.

Elias, M. E. (2016). La construcción de identidad profesional en los estudiantes del profesorado de educación primaria. Profesorado, 20, 335-365.

Edwards, F. C. E. y Edwards, R. J. (2016). A story of culture and teaching: the complexity of teacher identity formation. Curriculum Journal, 28(2), 190-211. doi $10.1080 / 09585176.2016 .1232200$

Fogle, L.W. y Moser, K. (2017). Language Teacher Identities in the Southern United States: Transforming Rural Schools. Journal of Language, Identity and Education, 16(2), 65-79. doi $10.1080 / 15348458.2016 .1277147$

Granado, C. y Puig, M. (2015). La identidad lectora de los maestros en formación como componente de su identidad docente. Un estudio de sus autobiografías como lectores. Ocnos, 13(11), 43-63. doi 10.18239/ocnos

Gu, M. y Benson, P. (2014). The formation of English teacher identities: A cross-cultural investigation. Language Teaching Research, 19(2), 187-206. doi 10.1177/1362168814541725

Hernández, Roberto et al. (2006). Metodología de la investigación. México. Mcgraw-Hill.

Hernández, R., Fernández, C. y Baptista, P. (2010). Metodología de la investigación. México: Mcgraw-Hill, Interamericana Editores.

Izadinia, M. (2016). Preservice teachers' professional identity development and the role of mentor teachers. International Journal of Mentoring and Coaching in Education, 5(2), 2-32.doi 10.1108/IJMCE-01-2016-0004

Jarauta, B. (2017). La construcción de la identidad profesional del maestro de primaria durante su formación inicial. El caso de la universidad de barcelona. Profesorado, 21, 103-122.

Kenny, A., Finneran, M. y Mitchell, E. (2015). Becoming an educator in and through the arts: Forming and informing emerging teachers' professional identity. Teaching and Teacher Education, 49, 159-167. doi 10.1016/j.tate.2015.03.004

Loewenson, R., Laurell, A. C., Hogstedt, C., D’Ambriosio, L., \& SHroff, Z. (2014). InvestigaciónAcción participativa en sistemas de salud, una guia de métodos.

Nghia, L. H., \& Tai, N. (2017). Preservice Teachers' Identity Development during the Teaching Internship. Australian Journal of Teacher Education, 42(428). doi 10.14221/ajte.2017v42n8.1

Nichols, S. L., Schutz, P. A., Rodgers, K., \& Bilica, K. (2017). Early career teachers' emotion and emerging teacher identities. Teachers and Teaching: Theory and Practice, 23(4), 406-421. doi 10.1080/13540602.2016.1211099

Peiteado, M. (2010). La institucionalización y marco legal de la formación inicial del profesorado de educación primaria y su impronta en los estilos de enseñanza. Innovación educativa, n.o 20, 145-156.

Pennington, M. C., \& Richards, J. C. (2015). Teacher Identity in Language Teaching: Integrating Personal, Contextual, and Professional Factors. RELC Journal, 47(1), 5-23. doi 10.1177/0033688216631219

Quilaqueo, D., Quintriqueo, S., \& Riquelme, E. (2016). Identidad Profesional Docente: Práctica Pedagógica en Contexto Mapuche. Estudios Pedagógicos, XLII No 2, 269-284. Doi 10.4067/S0718-07052016000200015

Quintriqueo, S. (2010). Implicancias de un modelo Curricular monocultural en contexto mapuche. Estudios pedagógicos. doi 10.4067/S0718-07052009000200010.

Rodríguez, G., Gil, J., \& García, E. (1996). Metodología de la Investigación Cualitativa. Málaga: Aljibe. 
Smilgienè, S. (2016). The Sociocultural Perspective on Non-native Teacher Identity Formation Process. Studies About Languages, 0(28), 67-79. Doi 10.5755/j01. sal.0.28.15129.

Taylor, S., \& Littleton, K. (2006). Biographies in talk: A narrative-discursive research approach. Qualitative Sociology Review, 2(1), 22-38.

Uitto, M., Kaunisto, S. L., Syrjälä, L., \& Estola, E. (2015). Silenced Truths: Relational and Emotional Dimensions of a Beginning Teacher's Identity as Part of the Micropolitical Context of School. Scandinavian Journal of Educational Research, 59(2), 162-176. doi 10.1080/00313831.2014.904414

Yuan, R., \& Burns, A. (2016). Theory and practice Teacher identity development through action research: a Chinese experience, 602(August). doi 10.1080/13540602.2016.1219713

Yazan, B. (2017). "It just made me look at language in a different way:" ESOL teacher candidates' identity negotiation through teacher education coursework. Linguistics and Education, 40, 38-49. doi.org/10.1016/j.linged.2017.06.002. 